Bull. Soc. math. France

129 (3), 2001, p. 361-377

\title{
PROPERTIES OF WIENER-WINTNER DYNAMICAL SYSTEMS
}

\author{
By I. ASSANi \& K. NiCOlaOU
}

\begin{abstract}
In this paper we prove the following results. First, we show the existence of Wiener-Wintner dynamical system with continuous singular spectrum in the orthocomplement of their respective Kronecker factors. The second result states that if $f \in L^{p}, p$ large enough, is a Wiener-Wintner function then, for all $\gamma \in\left(1+\frac{1}{2 p}-\frac{\beta}{2}, 1\right]$, there exists a set $X_{f}$ of full measure for which the series $\sum_{n=1}^{\infty} \frac{f\left(T^{n} x\right) e^{2 \pi i n \varepsilon}}{n^{\gamma}}$ converges uniformly with respect to $\varepsilon$.

RÉSumÉ (Propriétés des systèmes dynamiques de Wiener-Wintner)

Dans cette note nous démontrons les résultats suivants. Tout d'abord nous montrons l'existence de systèmes dynamiques ergodiques du type Wiener Wintner ayant un spectre singulier continu dans l'orthogonal de leur facteurs de Kronecker.Ensuite nous montrons que si $f \in L^{p}$ est une fonction du type Wiener-Wintner alors, pour $\gamma \in$ $\left(1+\frac{1}{2 p}-\frac{\beta}{2}, 1\right]$ on peut trouver un ensemble $X_{f}$ de mesure pleine pour lequel la série $\sum_{n=1}^{\infty} \frac{f\left(T^{n} x\right) e^{2 \pi i n \varepsilon}}{n^{\gamma}}$ converge uniformément en $\varepsilon$.
\end{abstract}

Texte reçu le 3 janvier 2000, révisé le 6 juillet 2000, accepté le 24 octobre 2000

I. Assani, Department of Mathematics, UNC Chapel Hill, NC 27599, USA

E-mail : assani@math.unc.edu

K. NiCOLAOU, Department of Mathematics, UNC Chapel Hill, NC 27599, USA

E-mail : katerina@math.unc.edu

2000 Mathematics Subject Classification. - 28D05, 11K38.

Key words and phrases. - Wiener Wintner dynamical systems, Wiener Wintner functions, Kronecker factor. 


\section{Introduction}

Let $(X, \mathcal{B}, \mu, T)$ be an ergodic dynamical system. Throughout this paper $\mathcal{K}$ will denote the Kronecker factor of $T$, i.e. the closed linear span in $L^{2}$ of the eigenfunctions for $T$. Wiener-Wintner functions and Wiener-Wintner dynamical systems were introduced and studied in [1] and [2].

Definition 1. - Let $(X, \mathcal{B}, \mu, T)$ be an ergodic dynamical system. A function $f$ is a Wiener-Wintner function of power type $\beta$ in $L^{1}$ if there exist finite positive constants $C_{f}$ and $\beta$ such that

$$
\left\|\sup _{\varepsilon}\left|\frac{1}{N} \sum_{n=1}^{N} f\left(T^{n} x\right) e^{2 \pi i n \varepsilon}\right|\right\|_{1} \leq \frac{C_{f}}{N^{\beta}}
$$

for all positive integers $N$.

Definition 2. - An ergodic dynamical system $(X, \mathcal{B}, \mu, T)$ is a WienerWintner dynamical system of power type $\beta$ in $L^{1}$ if there exists in $\mathcal{K}^{\perp}$ a dense set (for the $L^{2}$ norm) of Wiener-Wintner functions of power type $\beta$ in $L^{1}$.

In this paper whenever we say Wiener-Wintner we mean Wiener-Wintner of power type $\beta$ in $L^{1}$.

Among other properties these dynamical systems provide simpler proof of J. Bourgain [3] a.e. double recurrence result which answered a question of H. Furstenberg [4]. It was shown in [1] that $K$ automorphisms, product of $\mathrm{K}$ automorphism with any other Wiener-Wintner dynamical system, and factors of Wiener-Wintner dynamical systems are Wiener-Wintner dynamical systems. Discrete spectrum transformations are trivially Wiener-Wintner dynamical systems. It was also shown that for almost all irrational $\alpha$ (with respect to Lebesgue measure) the skew products $(x, y) \rightarrow(x+\alpha, y+x)$ on the 2 -Torus, $\mathbb{T}^{2}$, are Wiener-Wintner dynamical systems. This showed that there exist nontrivial Wiener-Wintner dynamical systems with zero entropy. All of the dynamical systems mentioned above have in fact a dense set of $L^{\infty}$ Wiener-Wintner functions. Note also that they all have Lebesgue spectrum in the orthocomplement of the Kronecker factor. This raises the question of finding an example of Wiener-Wintner dynamical systems with continuous singular spectrum in the orthocomplement of its Kronecker factor. We will show in this paper that the skew products $(x, y) \rightarrow(x+\alpha, y+\beta\{x\}), \alpha$ with unbounded partial quotients and $\beta$ irrational, on $\mathbb{T}^{2}$, provide us with examples of Wiener-Wintner dynamical systems with continuous singular spectrum in the othocomplement of the Kronecker factor. Hence, the Wiener-Wintner property of a dynamical system is not characterized by the nature of its spectrum in $\mathcal{K}^{\perp}$, (singular or Lebesgue).

The first author also showed that if $f$ is a Wiener-Wintner function, then there exists a set $X_{f}$ of full measure for which the rotated one sided ergodic

TOME $129-2001-\mathrm{N}^{\mathrm{O}} 3$ 
Hilbert transform for $f$, i.e. the series $\sum_{n=1}^{\infty} \frac{f\left(T^{n} x\right) e^{2 \pi i n \varepsilon}}{n}$, converges for all $\varepsilon$. Moreover, for $x \in X_{f}$ the map

$$
\varepsilon \longmapsto \sum_{n=1}^{\infty} \frac{f\left(T^{n} x\right) e^{2 \pi i n \varepsilon}}{n}
$$

is continuous $([1])$. In this paper we will study the convergence of the series $\sum_{n=1}^{\infty} \frac{f\left(T^{n} x\right) e^{2 \pi i n \varepsilon}}{n^{\gamma}}$ for $f \in L^{p}$ and $0<\gamma<1$. To get convergence and continuity in this case we will prove and use the property that

$$
\lim _{N \rightarrow \infty} \frac{1}{N^{\gamma \delta}} \sum_{n=\left[N^{\delta}\right]}^{\left[(N+1)^{\delta}\right]}|f|\left(T^{n} x\right)=0 \text { a.e. for } f \in L^{p}, 1 \leq p \leq \infty
$$

and for $\delta$ that depends on p. M. Schwartz showed that when $\gamma=\frac{\delta-1}{\delta}$ the result is not true. The averages $\frac{1}{N^{\delta}-1} \sum_{n=N^{\delta}}^{(N+1)^{\delta}} f\left(T^{n} x\right)$ will not converge a.e. even for characteristic functions $([10])$. We show that for $\gamma>1+\frac{1}{p}-\frac{1}{\delta}>1-\frac{1}{\delta}=\frac{\delta-1}{\delta}$,

$$
\frac{1}{N^{\gamma \delta}} \sum_{n=N^{\delta}}^{(N+1)^{\delta}}|f|\left(T^{n} x\right)
$$

converges to 0 . Note that convergence to zero is trivial for $f \in L^{\infty}$. In the case of $L^{\infty}$ i.i.d random variables it is easy to see that there exist WienerWintner functions, namely the Rademacher functions, for which the map $\varepsilon \mapsto$ $\sum_{n=1}^{\infty} \frac{f\left(T^{n} x\right) e^{2 \pi i n \varepsilon}}{n^{\gamma}}$ is not continuous if $\gamma \leq 1 / 2$.

Throughout this paper references about Diophantine approximations can be found in the classical book of A. Khinchin on continued fractions ([7]) or in [8].

\section{Existence of Wiener-Wintner dynamical systems with continuous singular spectrum in $\mathcal{K}^{\perp}$}

Let $\alpha$ be irrational and $\beta$ real. Let $T_{\alpha, \beta}: \mathbb{T}^{2} \rightarrow \mathbb{T}^{2}$ be the skew product defined on the 2-torus by

$$
T_{\alpha, \beta}(x, y)=(x+\alpha, y+\beta\{x\}) \bmod 1
$$

where $\{x\}$ is the fractional part of $\mathrm{x}$. i.e. $\{x\}=x \bmod 1$.

The case $\beta=1$ was studied in [1]. It was shown that for almost all irrational $\alpha$ the system $\left(\mathbb{T}^{2}, \mathcal{B}\left(\mathbb{T}^{2}\right), T_{\alpha, 1}, m\right)$ is a Wiener-Wintner dynamical system. These dynamical systems have Lebesgue spectrum in the orthocomplement of the Kronecker factor.

We will show that for all $\beta$ irrational there exists a set $\mathcal{I}_{\beta}$ of $\alpha$ with unbounded partial quotients of full measure such that for all $\alpha$ in $\mathcal{I}_{\beta}$ the system 
$\left(\mathbb{T}^{2}, \mathcal{B}\left(\mathbb{T}^{2}\right), T_{\alpha, \beta}, m\right)$ is a Wiener-Wintner dynamical system. In particular, the functions $f_{p, q}(x, y)=e^{2 \pi i p x} e^{2 \pi i q y}, q \neq 0$, form a dense set in $\mathcal{K}^{\perp}$ of WienerWintner functions. Such systems were shown in [9] to have continuous singular spectrum in the orthocomplement of the Kronecker factor. The results in [9] have been extended by A. Iwanik, M. Lemanczyk, and C. Mauduit in [5].

THEOREM 1. - For all $\beta \neq 0$ there exists a set $I_{\beta}$ of irrational $\alpha$ of full measure such that for all $\alpha$ in $I_{\beta}$ and for all $p, q(q \neq 0)$, we can find a constant $C_{q, \alpha, \beta}$ such that

$$
\sup _{\varepsilon}\left|\frac{1}{N} \sum_{n=1}^{N} f_{p, q}\left(T_{\alpha, \beta}^{n}(x, y)\right) e^{2 \pi i n \varepsilon}\right| \leq \frac{C_{q, \alpha, \beta}}{N^{s}}
$$

for some $s>0$, for all $(x, y)$ and for all positive integers $N$.

For the proof of this theorem we will use the following lemmas:

Lemma 1. - For all integers $m, m \neq 0$, and for all real numbers $\beta, \beta \neq 0$

$$
\int_{0}^{1} e^{2 \pi i \beta n\{m \alpha\}} d \alpha=\frac{1}{2 \pi i \beta n}\left(e^{2 \pi i \beta n}-1\right) .
$$

The proof of the above lemma is straightforward.

The following lemma can be extracted from the proof of the Proposition in [1].

LEMMA 2. - Let $\left\{f_{n}\right\}$ be a sequence of uniformly bounded functions. If

$$
\int_{0}^{1}\left|\frac{1}{N} \sum_{n=1}^{N} f_{n}(\alpha)\right| d \alpha \leq \frac{C}{N^{r}} \quad \text { for some } r>0,
$$

then for almost every $\alpha$,

$$
\left|\frac{1}{N} \sum_{n=1}^{N} f_{n}(\alpha)\right| \leq \frac{C_{r, \alpha}}{N^{\rho_{r}}},
$$

where $C_{r, \alpha}$ is a new positive constant that depends on $r$ and $\alpha$, and $\rho_{r}$ is a positive constant less than $r$ that depends on $r$.

Proof. - Without loss of generality, we can assume that $\left\{f_{n}\right\}$ is uniformly bounded by 1 .

We will first prove the result for a subsequence, and then for the sequence itself. Let $0<s<r$. Then,

$$
\int_{0}^{1}\left[N^{s}\left|\frac{1}{N} \sum_{n=1}^{N} f_{n}(\alpha)\right|\right] d \alpha \leq \frac{C}{N^{r-s}} .
$$

TOME $129-2001-\mathrm{N}^{\mathrm{O}} 3$ 
Let $N=M^{l}$ for 1 large enough. That is, $l(r-s)>1$. This implies that

$$
\sum_{M=1}^{\infty} \int\left|\left(M^{l}\right)^{s} \frac{1}{M^{l}} \sum_{n=1}^{M^{l}} f_{n}(\alpha)\right| d \alpha \leq \sum_{M=1}^{\infty} \frac{C}{M^{l(r-s)}}<\infty .
$$

Hence, for a.e. $\alpha$

$$
\left|\frac{1}{M^{l}} \sum_{n=1}^{M^{l}} f_{n}(\alpha)\right| \leq \frac{C_{\alpha}}{\left(M^{l}\right)^{s}} .
$$

Now, let $M^{l} \leq N \leq(M+1)^{l}$. Then,

$$
\left|\frac{1}{N} \sum_{n=1}^{N} f_{n}(\alpha)\right| \leq\left|\frac{1}{M^{l}} \sum_{n=1}^{M^{l}} f_{n}(\alpha)\right|+\frac{1}{M^{l}} \sum_{n=M^{l}+1}^{(M+1)^{l}}\left|f_{n}(\alpha)\right| .
$$

The first term is less than or equal to $C_{\alpha} /\left(M^{l}\right)^{s}$ for a.e. $\alpha$. The second term

$$
\begin{aligned}
\frac{1}{M^{l}} \sum_{n=M^{l}+1}^{(M+1)^{l}}\left|f_{n}(\alpha)\right| & \leq \frac{(M+1)^{l}-M^{l}}{M^{l}} \text { since }\left|f_{n}(\alpha)\right| \leq 1 \text { for all } \alpha \text { and for all } n \\
& \leq \frac{C M^{l-1}}{M^{l}}=\frac{C}{M} .
\end{aligned}
$$

Hence, for a.e. $\alpha$,

$$
\left|\frac{1}{N} \sum_{n=1}^{N} f_{n}(\alpha)\right| \leq \frac{C_{\alpha}}{M^{l s}}+\frac{C}{M} \leq \frac{C_{\alpha}}{M^{\gamma}},
$$

where $\gamma=\min \{l s, 1\}$. Note that

$$
\frac{1}{M^{\gamma}}=\left(\frac{M+1}{M}\right)^{\gamma} \frac{1}{(M+1)^{\gamma}} \leq 2^{\gamma} \frac{1}{(M+1)^{\gamma}} \leq \frac{2^{\gamma}}{N^{\gamma / l}} .
$$

Letting $\rho_{r}=\gamma / l$ we have the desired result.

LEMMA 3. - Let $f_{h}(x):[0,1) \rightarrow S^{1}=\{z \in \mathbb{C}:|z|=1\}$ be the function defined by

$$
f_{h}(x)=e^{2 \pi i q \beta(\{x\}+\{x+\alpha\}+\cdots+\{x+(h-1) \alpha\})}
$$

where $\alpha$ is a fixed irrational number and $\beta \in \mathbb{R} \backslash\{0\}$. Then $f_{h}$ has bounded variation, $V\left(f_{h}\right)$, and $V\left(f_{h}\right)=O(h)$.

Proof. - This follows directly from the inequality $V(f g) \leq V(f)+V(g)$ for $f$ and $g$ such that $|f| \equiv|g| \equiv 1$.

LEMMA 4. - There exists a set $I_{\beta}$ of irrational $\alpha$ of full measure such that for $\alpha$ in $I_{\beta}$ we can find a constant $C_{\alpha}$ and a number $s, 0<s<1$, such that

$$
\left|\int_{0}^{1} f_{h}(t) d t\right|=\left|\int_{0}^{1} e^{2 \pi i q \beta(\{t\}+\{t+\alpha\}+\cdots+\{t+(h-1) \alpha\})} d t\right| \leq \frac{C_{\alpha}}{h^{s}} .
$$

BULLETIN DE LA SOCiÉtÉ MATHÉMATIQUE DE FRANCE 
Proof. - It was shown in [9] that

$$
\int_{0}^{1} f_{h}(t) d t=\frac{C e^{2 \pi i q h \beta}\left(e^{2 \pi i q \beta}-1\right)}{2 \pi i q h \beta} \sum_{j=1}^{h} e^{-2 \pi i q h \beta\left\{l_{j} \alpha\right\}} e^{-2 \pi i q \beta j}
$$

where $C=\prod_{m=0}^{h-1} e^{2 \pi i q \beta\{m \alpha\}}$ and $\left\{1-\left\{l_{j} \alpha\right\}\right\}_{j=1}^{h}$ is the set of discontinuities of $f_{h}$ arranged in increasing order with $l_{h}=0$. Hence,

$$
\left|\int_{0}^{1} f_{h}(t) d t\right| \leq \frac{1}{\pi q \beta}\left|\frac{1}{h} \sum_{j=1}^{h} e^{-2 \pi i q h \beta\left\{l_{j} \alpha\right\}} e^{-2 \pi i q \beta j}\right| .
$$

We will use Van der Corput's inequality (see [8] for a proof). Recall that this inequality says the following: If $\left(u_{n}\right)_{0 \leq n \leq N-1}$ is a family of complex numbers and if $\mathrm{H}$ is an integer between 0 and $\bar{N}-1$, then

$$
\begin{aligned}
& \left|\frac{1}{N} \sum_{n=0}^{N-1} u_{n}\right|^{2} \\
\leq & \frac{N+H}{N^{2}(H+1)} \sum_{n=0}^{N-1}\left|u_{n}\right|^{2}+\frac{2(N+H)}{N^{2}(H+1)^{2}} \sum_{h=1}^{H}(H+1-h) \operatorname{Re}\left(\sum_{n=0}^{N-h-1} \overline{u_{n+h}} u_{n}\right) .
\end{aligned}
$$

We have then

$$
\begin{aligned}
& \left|\frac{1}{h} \sum_{j=1}^{h} e^{-2 \pi i q h \beta\left\{l_{j} \alpha\right\}} e^{-2 \pi i q \beta j}\right|^{2} \leq \frac{h+K}{h^{2}(K+1)} \sum_{j=0}^{h-1} 1 \\
& +\frac{2(h+K)}{h^{2}(K+1)^{2}} \sum_{r=1}^{K}(K+1-r) \cdot \\
& \quad \cdot \operatorname{Re}\left(\sum_{j=0}^{h-r-1} e^{-2 \pi i q \beta h\left\{l_{j+r+1} \alpha\right\}} e^{-2 \pi i q \beta(j+r+1)} e^{2 \pi i q \beta h\left\{l_{j+1} \alpha\right\}} e^{2 \pi i q \beta(j+1)}\right) \\
& \leq \frac{2}{K+1}+\frac{2(h+K)}{h^{2}(K+1)^{2}} \sum_{r=1}^{K}(K+1-r) \cdot \\
& \left.=\frac{2}{K+1}+\frac{2(h+K)}{h^{2}(K+1)^{2}} \sum_{r=1}^{K}(K+1-r) \cdot \operatorname{Re} e^{-2 \pi i q \beta r} \sum_{j=0}^{h-r-1} e^{2 \pi i q \beta h\left(\left\{l_{j+1} \alpha\right\}-\left\{l_{j+r+1} \alpha\right\}\right)}\right] \\
& \cdot \operatorname{Re}\left[e^{-2 \pi i q \beta r} \sum_{j=0}^{h-r-1} e^{2 \pi i q \beta h\left\{\left(l_{j+1}-l_{j+r+1}\right) \alpha\right\}}\right]
\end{aligned}
$$

since $\left\{l_{j+1} \alpha\right\}>\left\{l_{j+r+1} \alpha\right\}$.

TOME $129-2001-\mathrm{N}^{\mathrm{O}} 3$ 
Integrating with respect to $\alpha$ and using Lemma 1 we get that

$$
\begin{aligned}
\int_{0}^{1} \mid & \left.\frac{1}{h} \sum_{j=1}^{h} e^{-2 \pi i q \beta h\left\{l_{j} \alpha\right\}} e^{-2 \pi i q \beta j}\right|^{2} d \alpha \\
& \leq \frac{2}{K+1}+\frac{2(h+K)}{h^{2}(K+1)^{2}} \sum_{r=1}^{K}(K-1-r) \operatorname{Re}\left[e^{-2 \pi i q \beta r} \sum_{j=0}^{h-r-1} \frac{e^{2 \pi i q \beta h}-1}{2 \pi i q \beta h}\right] \\
& \leq \frac{2}{K+1}+\frac{2(h+K)}{h^{2}(K+1)^{2}} K \sum_{r=1}^{K}\left|(h-r) \frac{e^{-2 \pi i q \beta r}\left(e^{2 \pi i q \beta h}-1\right)}{2 \pi i q \beta h}\right| \\
& \leq \frac{2}{K+1}+\frac{2(h+K)}{h^{2}(K+1)^{2}} \frac{K^{2}}{\pi q \beta} .
\end{aligned}
$$

By letting $K=h-1$ we have

$$
\int_{0}^{1}\left|\frac{1}{h} \sum_{j=1}^{h} e^{-2 \pi i q \beta h\left\{\left\{l_{j} \alpha\right\}\right\}} e^{-2 \pi i q \beta j}\right|^{2} d \alpha \leq \frac{C}{h}
$$

where $\mathrm{C}$ depends on $q$ and $\beta$. By Lemma 2 , there exists a set $I_{\beta}$ of irrational $\alpha$ of full measure such that for $\alpha$ in $I_{\beta}$ we can find a constant $C_{\alpha}$ and a number $s, 0<s<1 / 2<1$, such that

$$
\left|\frac{1}{h} \sum_{j=1}^{h} e^{-2 \pi i q \beta h\left\{l_{j} \alpha\right\}} e^{-2 \pi i q \beta j}\right| \leq \frac{C_{\alpha}}{h^{s}} .
$$

So, from (1) we can conclude that

$$
\left|\int_{0}^{1} f_{h}(t) d t\right| \leq \frac{C_{\alpha}}{h^{s}}
$$

where the constant $C_{\alpha}$ also depends on $q$ and $\beta$.

\section{Proof of Theorem 1}

$$
\begin{aligned}
\sup _{\varepsilon} \mid \frac{1}{N} \sum_{n=1}^{N} f_{p, q} & \left(T_{\alpha, \beta}^{n}(x, y)\right) e^{2 \pi i n \varepsilon} \mid \\
& =\sup _{\varepsilon}\left|\frac{1}{N} \sum_{n=1}^{N} e^{2 \pi i p(x+n \alpha)} e^{2 \pi i q(y+\beta\{x\}+\cdots+\{x+(n-1) \alpha\})} e^{2 \pi i n \varepsilon}\right| \\
& =\sup _{\varepsilon}\left|\frac{1}{N} \sum_{n=1}^{N} e^{2 \pi i q \beta(\{x\}+\cdots+\{x+(n-1) \alpha\})} e^{2 \pi i n \varepsilon}\right| .
\end{aligned}
$$

We will use Van Der Corput's inequality to estimate

$$
\sup _{\varepsilon}\left|\frac{1}{N} \sum_{n=1}^{N} e^{2 \pi i q \beta(\{x\}+\cdots+\{x+(n-1) \alpha\})} e^{2 \pi i n \varepsilon}\right|^{2}
$$

BULLETIN DE LA SOCIÉTÉ MATHÉMATIQUE DE FRANCE 
Let $f_{h}(x)=e^{2 \pi i q \beta(\{x\}+\{x+\alpha\}+\cdots+\{x+(h-1) \alpha\})}$. Then,

$$
\begin{aligned}
& \sup _{\varepsilon}\left|\frac{1}{N} \sum_{n=1}^{N} e^{2 \pi i q \beta(\{x\}+\cdots+\{x+(n-1) \alpha\})} e^{2 \pi i n \varepsilon}\right|^{2} \\
& \leq \frac{N+H}{N^{2}(H+1)} \sum_{n=0}^{N-1} 1+\frac{2(N+H)}{N^{2}(H+1)^{2}} \sum_{h=1}^{H}(H+1-h) \cdot \\
& \leq \frac{2}{H+1}+\frac{4}{(H+1)^{2}} \sum_{h=1}^{N-h-1} f_{n+h+2}(H) \overline{f_{n+1}(x)} \mid \\
&=\frac{2}{H+1}+\frac{4}{(H+1)^{2}} \sum_{h=1}^{N-h-1}(H+1-h) \cdot
\end{aligned}
$$

We will focus our attention on $\left|\frac{1}{N} \sum_{n=1}^{N-h} f_{h}(\{x+n \alpha\})\right|$. By Lemma $3 f_{h}$ has bounded variation. Hence, by Koksma's inequality ([8], p. 143)

$$
\left|\frac{1}{N} \sum_{n=1}^{N} f_{h}(\{x+n \alpha\})-\int_{0}^{1} f_{h}(t) d t\right| \leq V\left(f_{h}\right) D_{N}(\{x+n \alpha\})
$$

where $D_{N}(\{x+n \alpha\})$ denotes the discrepancy of the sequence $\{\{x+n \alpha\}\}_{n=1}^{N}$. $D_{N}$ is as defined in [8], p. 88. Namely,

$$
\begin{aligned}
D_{N}=D_{N}\left(x_{1}, \cdots, x_{N}\right) & \\
& =\sup _{0 \leq \alpha<\beta \leq 1}\left|\frac{\#\left\{n: 1 \leq n \leq N \text { and } x_{n} \in[\alpha, \beta)\right\}}{N}-(\beta-\alpha)\right| .
\end{aligned}
$$

So, from (2) we have that

$$
\begin{aligned}
& \left|\frac{1}{N} \sum_{n=0}^{N-h-1} f_{h}(\{x+(n+1) \alpha\})\right| \\
& \leq\left|\frac{1}{N} \sum_{n=1}^{N} f_{h}(\{x+n \alpha\})\right|+\left|\frac{1}{N} \sum_{n=N-h}^{N} f_{h}(\{x+(n+1) \alpha\})\right| \\
& \leq\left|\int_{0}^{1} f_{h}(t) d t\right|+V\left(f_{h}\right) D_{N}(\{x+n \alpha\})+\frac{h}{N}
\end{aligned}
$$

TOME $129-2001-\mathrm{N}^{\mathrm{O}} 3$ 
By Lemma 4,

for almost all $\alpha$ and for some $0<s<1$.

$$
\left|\int_{0}^{1} f_{h}(t) d t\right| \leq \frac{C_{\alpha}}{h^{s}}
$$

Now, we need an estimate for $D_{N}(\{x+n \alpha\})$. We know from Khinchin's inequality $([6])$, see also [8], that for every positive nondecreasing function $\mathrm{g}$ such that $\sum_{n=1}^{\infty} \frac{1}{g(n)}<\infty$ the discrepancy $D_{N}(\{n \alpha\})$ satisfies

$$
N D_{N}(\{n \alpha\})=O((\log N) g(\log \log N))
$$

for almost all $\alpha$. By letting $g(n)=n^{2}$ we get that $D_{N}(\{n \alpha\}) \leq C_{\alpha^{\prime}} / N^{s^{\prime}}$ for almost all $\alpha^{\prime}$ and for $0<s^{\prime}<1$. Since discrepancy is translation mod 1 invariant,

$$
D_{N}(\{x+n \alpha\}) \leq \frac{C_{\alpha^{\prime}}}{N^{s^{\prime}}}
$$

for all $x$, for almost all $\alpha^{\prime}$, and for $0<s^{\prime}<1$.

Going back to (3), we now have

$$
\begin{aligned}
\left|\frac{1}{N} \sum_{n=0}^{N-h-1} f_{h}(\{x+(n+1) \alpha\})\right| & \leq\left|\int_{0}^{1} f_{h}(t) d t\right|+V\left(f_{h}\right) D_{N}(\{x+n \alpha\})+\frac{h}{N} \\
& \leq \frac{C_{\alpha}}{h^{s}}+V\left(f_{h}\right) \frac{C_{\alpha}}{N^{s^{\prime}}}+\frac{h}{N}
\end{aligned}
$$

for all $x$, for almost all $\alpha$, and for some positive constants $s, s^{\prime}<1$. Furthermore, by Lemma $3, V\left(f_{h}\right)=O(h)$ which implies

$$
\left|\frac{1}{N} \sum_{n=0}^{N-h-1} f_{h}(\{x+(n+1) \alpha\})\right| \leq \frac{C_{\alpha}}{h^{s}}+\frac{h C_{\alpha}}{N^{s^{\prime}}}+\frac{h}{N} .
$$

Hence,

$$
\begin{aligned}
\sup _{\varepsilon} \mid & \left.\frac{1}{N} \sum_{n=1}^{N} e^{2 \pi i q \beta(\{x\}+\cdots+\{x+(n-1) \alpha\})} e^{2 \pi i n \varepsilon}\right|^{2} \\
& \leq \frac{2}{H+1}+\frac{4}{(H+1)^{2}} \sum_{h=1}^{H}(H+1-h)\left|\frac{1}{N} \sum_{n=0}^{N-h-1} f_{h}(\{x+(n+1) \alpha\})\right| \\
& \leq \frac{2}{H+1}+\frac{4}{H} \sum_{h=1}^{H}\left(\frac{C_{\alpha}}{h^{s}}+\frac{h C_{\alpha}}{N^{s^{\prime}}}+\frac{h}{N}\right) \\
& \leq \frac{2}{H+1}+\frac{4 H^{1-s} C_{\alpha}}{H}+\frac{4 H C_{\alpha}}{N^{s^{\prime}}}+\frac{4 H}{N} \\
& =\frac{2}{H+1}+\frac{4 C_{\alpha}}{H^{s}}+\frac{4 H C_{\alpha}}{N^{s^{\prime}}}+\frac{4 H}{N}
\end{aligned}
$$

By letting $H=\left[N^{s^{\prime} / 2}\right]$ we get the desired result.

BULLETIN DE LA SOCIÉTÉ MATHÉMATIQUE DE FRANCE 


\section{REMARK}

(1) It follows easily from the estimates in the above proof that for all $\beta \neq 0$ there exists a set $I_{\beta}$ of irrational $\alpha$ such that for all $\alpha \in I_{\beta}$ the transformation $T_{\alpha, \beta}$ is ergodic.

(2) We can remark that the inequality in Theorem 1 is uniform in $(x, y)$. This makes $f_{p, q}$ Wiener-Wintner functions of power type in $L^{\infty}([2])$.

By using only Van Der Corput's inequality we can also get the following:

THEOREM 2. - If $\alpha$ is of finite type $\eta$, then the system $\left(\mathbb{T}, \mathcal{B}, T_{\alpha, 1}, m\right)$ is a Wiener-Wintner dynamical system.

Proof. — We show that the functions $f_{p, q}, q \neq 0$, are Wiener-Wintner functions. As in [1]

$$
\begin{aligned}
\sup _{\varepsilon} \mid \frac{1}{N} \sum_{n=1}^{N} & \left.f_{p, q}\left(T_{\alpha, 1}^{n}(x, y)\right) e^{2 \pi i n \varepsilon}\right|^{2} \\
& \leq \frac{2}{H+1}+\frac{4}{(H+1)^{2}} \sum_{h=1}^{H}(H+1-h)\left|\frac{1}{N} \sum_{n=1}^{N-h-1} e^{-2 \pi i q h n \alpha}\right| \\
& \leq \frac{2}{H+1}+\frac{4}{H+1} \sum_{h=1}^{H}\left|\frac{1}{N} \sum_{n=1}^{N} e^{-2 \pi i q h n \alpha}-\frac{1}{N} \sum_{n=N-h}^{N} e^{-2 \pi i q h n \alpha}\right| \\
& \leq \frac{2}{H+1}+\frac{4 H}{N}+\frac{4}{H} \sum_{h=1}^{H}\left|\frac{1}{N} \sum_{n=1}^{N} e^{-2 \pi i q h n \alpha}\right|
\end{aligned}
$$

Now,

$$
\begin{aligned}
\frac{4}{H} \sum_{h=1}^{H}\left|\frac{1}{N} \sum_{n=1}^{N} e^{-2 \pi i q h n \alpha}\right|=\frac{4}{H} \sum_{h=1}^{H} \frac{1}{N}\left|\frac{\sin (\pi q h N \alpha)}{\sin (\pi q h \alpha)}\right| & \leq \frac{4}{H} \sum_{h=1}^{H} \frac{1}{N}\left|\frac{1}{\sin (\pi q h \alpha)}\right| \\
& =\frac{4}{H} \sum_{h=1}^{H} \frac{1}{N} \frac{1}{\sin (\pi\langle q h \alpha\rangle)} .
\end{aligned}
$$

Let $\gamma$ be any number less than 1 . Then,

(4) $\frac{4}{H} \sum_{h=1}^{H} \frac{1}{N} \frac{1}{\sin (\pi\langle q h \alpha\rangle)}=\frac{4}{H} \sum_{\left\{h:\langle q h \alpha\rangle \leq 1 / N^{\gamma}\right\}} \frac{1}{N} \frac{1}{\sin (\pi\langle q h \alpha\rangle)}$

$$
+\frac{4}{H} \sum_{\left\{h:\langle q h \alpha\rangle>1 / N^{\gamma}\right\}} \frac{1}{N} \frac{1}{\sin (\pi\langle q h \alpha\rangle)} .
$$

TOME $129-2001-\mathrm{N}^{\mathrm{O}} 3$ 
If $\langle q h \alpha\rangle \leq 1 / N^{\gamma}$ and $N$ is large enough, then $\sin (\pi\langle q h \alpha\rangle) \geq \frac{\pi}{2}\langle q h \alpha\rangle$. So,

$$
\begin{aligned}
\frac{4}{H} \sum_{\left\{h:\langle q h \alpha\rangle \leq 1 / N^{\gamma}\right\}} \frac{1}{N} \frac{1}{\sin (\pi\langle q h \alpha\rangle)} & \leq \frac{8}{H} \sum_{h=1}^{H} \frac{1}{N} \frac{1}{\pi\langle q h \alpha\rangle} \\
& \leq \frac{8 q}{N \pi} \sum_{h=1}^{H} \frac{1}{q h\langle q h \alpha\rangle} .
\end{aligned}
$$

It was shown in [8], p. 123, that if $\alpha$ is of finite type $\eta$, then

$$
\sum_{n=1}^{N} \frac{1}{n\langle n \alpha\rangle}=O\left(N^{\eta-1+\varepsilon}\right) \quad \text { for all } \varepsilon>0 .
$$

We can conclude that

$$
\frac{4}{H} \sum_{\left\{h:\langle q h \alpha\rangle \leq 1 / N^{\gamma}\right\}} \frac{1}{N} \frac{1}{\sin (\pi\langle q h \alpha\rangle)} \leq \frac{C_{\varepsilon} H^{\eta-1+\varepsilon}}{N} .
$$

If $\langle q h \alpha\rangle>1 / N^{\gamma}$, then $\sin (\pi\langle q h \alpha\rangle)>\sin \left(\pi / N^{\gamma}\right)$ since $\langle q h \alpha\rangle<1 / 2$. This implies that

(6) $\frac{4}{H} \sum_{\left\{h:\langle q h \alpha\rangle>1 / N^{\gamma}\right\}} \frac{1}{N} \frac{1}{\sin (\pi\langle q h \alpha\rangle)} \leq \frac{4}{H} \sum_{h=1}^{H} \frac{1}{N} \frac{1}{\sin \left(\pi / N^{\gamma}\right)} \leq \frac{C^{\prime}}{N} N^{\gamma}=\frac{C^{\prime}}{N^{s^{\prime}}}$ for $\mathrm{N}$ large and $s^{\prime}=1-\gamma>0$.

From (4),(5), and (6) we now have

$$
\frac{4}{H} \sum_{h=1}^{H} \frac{1}{N} \frac{1}{\sin (\pi\langle q h \alpha\rangle)} \leq \frac{C_{\varepsilon} H^{\eta-1+\varepsilon}}{N}+\frac{C^{\prime}}{N^{s^{\prime}}} .
$$

Hence,

$$
\sup _{\varepsilon}\left|\frac{1}{N} \sum_{n=1}^{N} f_{p, q}\left(T_{\alpha, 1}^{n}(x, y)\right) e^{2 \pi i n \varepsilon}\right|^{2} \leq \frac{2}{H}+\frac{4 H}{N}+\frac{C_{\varepsilon} H^{\eta-1+\varepsilon}}{N}+\frac{C^{\prime}}{N^{s^{\prime}}} .
$$

Let $H=\left[N^{r}\right]$ for some $0<r<\min \left\{1, \frac{1}{\eta-1+\varepsilon}\right\}$. Then,

$$
\begin{aligned}
& \int_{0}^{1} \int_{0}^{1} \sup _{\varepsilon} \mid \frac{1}{N} \sum_{n=1}^{N} f_{p, q}\left(T_{\alpha, 1}^{n}(x, y)\right) e^{2 \pi i n \varepsilon} \mid d x d y \\
& \leq\left[\int_{0}^{1} \int_{0}^{1} \sup _{\varepsilon}\left|\frac{1}{N} \sum_{n=1}^{N} f_{p, q}\left(T_{\alpha, 1}^{n}(x, y)\right) e^{2 \pi i n \varepsilon}\right|^{2} d x d y\right]^{1 / 2} \leq \frac{C}{N^{t}},
\end{aligned}
$$

for some $0<t<1$.

BULletin DE LA SOCIÉtÉ MATHÉMATIQUE DE FRANCE 


\section{Convergence of the rotated fractional one sided ergodic Hilbert transform}

The following theorem was proved for the case $\gamma=1$ and $f \in L^{2}$ in [1].

Theorem 3. - Let $(X, \mathcal{B}, \mu, T)$ be an ergodic dynamical system. Let $f \in \mathcal{K}^{\perp}$ be a Wiener-Wintner function such that

$$
\left\|\sup _{\varepsilon}\left|\frac{1}{N} \sum_{n=1}^{N} f\left(T^{n} x\right) e^{2 \pi i n \varepsilon}\right|\right\|_{1} \leq \frac{C_{f}}{N^{\beta}}
$$

where $C_{f}, \beta$ are positive constants with $\beta<1$. Let $p \geq \max \{2,1 / \beta\}$. If $1+$ $\frac{1}{2 p}-\frac{\beta}{2}<\gamma \leq 1$ and $f \in L^{p}$, then there exists a set $X_{f}$ of full measure such that for all $x \in X_{f}$ the series $\sum_{n=1}^{\infty} \frac{f\left(T^{n} x\right) e^{2 \pi i n \varepsilon}}{n^{\gamma}}$ converges for all $\varepsilon$. Furthermore, the map

$$
\varepsilon \longmapsto \sum_{n=1}^{\infty} \frac{f\left(T^{n} x\right) e^{2 \pi i n \varepsilon}}{n^{\gamma}}
$$

is continuous for all $x \in X_{f}$.

To prove the above theorem, we will use the following proposition.

Proposition 1. - Let $f \in L^{p}, 1 \leq p \leq \infty$, be a positive function. Let $\gamma$ be such that $0<\gamma \leq 1$. Let $\delta>0$ be such that $1<\delta<1 /\left(1+\frac{1}{p}-\gamma\right)$. (Note that $\delta<1 /(1-\gamma) \Rightarrow \gamma>(\delta-1) / \delta)$. Then,

$$
\lim _{N \rightarrow \infty} \frac{1}{N^{\gamma \delta}} \sum_{n=\left[N^{\delta}\right]}^{\left[(N+1)^{\delta}\right]} f\left(T^{n} x\right)=0 \text { a.e. }
$$

Proof. - The result is trivial if $p=\infty$. If $p \neq \infty$, then we proceed as follows: Let $g_{n}=f \circ T^{n}-f \circ T^{n} \wedge n^{1 / p}$. Then,

$$
\frac{1}{N^{\gamma \delta}} \sum_{n=\left[N^{\delta}\right]}^{\left[(N+1)^{\delta}\right]} f\left(T^{n} x\right)=\frac{1}{N^{\gamma \delta}} \sum_{n=\left[N^{\delta}\right]}^{\left[(N+1)^{\delta}\right]} f\left(T^{n} x\right) \wedge n^{1 / p}+\frac{1}{N^{\gamma \delta}} \sum_{n=\left[N^{\delta}\right]}^{\left[(N+1)^{\delta}\right]} g_{n}(x) .
$$

First, we will show the a.e. convergence to zero of the second term:

$$
\begin{aligned}
\mu\left(\left\{x: \sum_{n=\left[N^{\delta}\right]}^{\left[(N+1)^{\delta}\right]} g_{n}(x)>N^{\gamma \delta} \varepsilon\right\}\right) & \leq \mu\left(\left\{x: \bigcup_{n=\left[N^{\delta}\right]}^{\left[(N+1)^{\delta}\right]} g_{n}(x)>0\right\}\right) \\
& \leq \sum_{n=\left[N^{\delta}\right]}^{\left[(N+1)^{\delta}\right]} \mu\left(\left\{x: f^{p}(x)>n\right\}\right),
\end{aligned}
$$

since $\mathrm{T}$ is measure preserving.

TOME $129-2001-\mathrm{N}^{\mathrm{O}} 3$ 
Now,

$$
\begin{aligned}
\sum_{N=1}^{\infty} \mu\left(\left\{x: \sum_{n=\left[N^{\delta}\right]}^{\left[(N+1)^{\delta}\right]} g_{n}(x)>N^{\gamma \delta} \varepsilon\right\}\right) & \leq \sum_{N=1}^{\infty} \sum_{n=\left[N^{\delta}\right]}^{\left[(N+1)^{\delta}\right]} \mu\left(\left\{x: f^{p}(x)>n\right\}\right) \\
& =\sum_{N=1}^{\infty} \mu\left(\left\{x: f^{p}(x)>N\right\}\right) \\
& \leq \int_{0}^{\infty} \mu\left(\left\{x: f^{p}(x)>t\right\}\right) d t=\|f\|_{p}^{p}<\infty
\end{aligned}
$$

Hence, by the Borel-Cantelli Lemma,

$$
\mu\left(\varlimsup_{N}\left\{x: \sum_{n=\left[N^{\delta}\right]}^{\left[(N+1)^{\delta}\right]} g_{n}(x)>N^{\gamma \delta} \varepsilon\right\}\right)=0 \quad \text { for all } \varepsilon .
$$

So, we can conclude that

$$
\lim _{N \rightarrow \infty} \frac{1}{N^{\gamma \delta}} \sum_{n=\left[N^{\delta}\right]}^{\left[(N+1)^{\delta}\right]} g_{n}(x)=0 \text { a.e., }
$$

as desired, since $g_{n}$ is positive.

To finish the proof of the proposition, it remains to show that

$$
\frac{1}{N^{\gamma \delta}} \sum_{n=\left[N^{\delta}\right]}^{\left[(N+1)^{\delta}\right]} f\left(T^{n} x\right) \wedge n^{1 / p}
$$

approaches 0 a.e. as $N \rightarrow \infty$. But,

$$
\begin{aligned}
\frac{1}{N^{\gamma \delta}} \sum_{n=\left[N^{\delta}\right]}^{\left[(N+1)^{\delta}\right]} f\left(T^{n} x\right) & \wedge n^{1 / p} \leq \frac{1}{N^{\gamma \delta}} \sum_{n=\left[N^{\delta}\right]}^{\left[(N+1)^{\delta}\right]} n^{1 / p} \\
& \leq \frac{1}{N^{\gamma \delta}} \int_{n=\left[N^{\delta}\right]}^{\left[(N+1)^{\delta}\right]+1} x^{1 / p} d x \\
& =\frac{1}{N^{\gamma \delta}}\left(\frac{1}{\frac{1}{p}+1}\right)\left\{\left(\left[(N+1)^{\delta}\right]+1\right)^{\frac{1}{p}+1}-\left[N^{\delta}\right]^{\frac{1}{p}+1}\right\} \\
& \leq \frac{1}{N^{\gamma \delta}}\left(\frac{1}{\frac{1}{p}+1}\right)\left\{\left((N+1)^{\delta}+1\right)^{\frac{1}{p}+1}-\left(N^{\delta}-1\right)^{\frac{1}{p}+1}\right\} \\
& \leq C \frac{N^{\delta\left(\frac{1}{p}+1\right)-1}}{N^{\gamma \delta}} \text { since } \delta>1 \\
& =C \frac{1}{N^{\gamma \delta-\delta\left(\frac{1}{p}+1\right)+1}} \rightarrow 0
\end{aligned}
$$

since by assumption $\gamma \delta-\delta\left(\frac{1}{p}+1\right)+1>0$.

BULletin DE LA SOCiÉtÉ MATHÉMATIQUE DE FRANCE 
Proof of Theorem 3. - Let

$$
M_{N}^{\varepsilon}(f)=\frac{1}{N} \sum_{n=1}^{N} f\left(T^{n} x\right) e^{2 \pi i n \varepsilon}, \quad S_{N}^{\varepsilon}=\sum_{n=1}^{N} \frac{f\left(T^{n} x\right) e^{2 \pi i n \varepsilon}}{n^{\gamma}} .
$$

Then,

(7) $S_{M}^{\varepsilon}(f)-S_{N}^{\varepsilon}(f)=\sum_{n=N+1}^{M} \frac{n M_{n}^{\varepsilon}(f)-(n-1) M_{n-1}^{\varepsilon}(f)}{n^{\gamma}}$

$$
\begin{aligned}
& =\sum_{n=N+1}^{M} \frac{n M_{n}^{\varepsilon}(f)}{n^{\gamma}}-\sum_{n=N+1}^{M} \frac{(n-1) M_{n-1}^{\varepsilon}(f)}{n^{\gamma}} \\
& =\sum_{n=N+1}^{M} \frac{n M_{n}^{\varepsilon}(f)}{n^{\gamma}}-\sum_{n=N}^{M-1} \frac{n M_{n}^{\varepsilon}(f)}{(n+1)^{\gamma}} \\
& =\sum_{n=N+1}^{M-1} n M_{n}^{\varepsilon}(f)\left(\frac{1}{n^{\gamma}}-\frac{1}{(n+1)^{\gamma}}\right)+\frac{M M_{M}^{\varepsilon}(f)}{M^{\gamma}}-\frac{N M_{N}^{\varepsilon}(f)}{(N+1)^{\gamma}} .
\end{aligned}
$$

So, we have

$$
\begin{aligned}
\left\|\sup _{\varepsilon}\left|S_{M}^{\varepsilon}(f)-S_{N}^{\varepsilon}(f)\right|\right\|_{1} & \leq \sum_{N+1}^{M-1}\left[\frac{1}{n^{\gamma}}-\frac{1}{(n+1)^{\gamma}}\right]\left\|\sup _{\varepsilon}\left|n M_{n}^{\varepsilon}(f)\right|\right\|_{1} \\
& +\frac{M}{M^{\gamma}}\left\|\sup _{\varepsilon}\left|M_{M}^{\varepsilon}(f)\right|\right\|_{1}+\frac{N}{(N+1)^{\gamma}}\left\|\sup _{\varepsilon}\left|M_{N}^{\varepsilon}(f)\right|\right\|_{1} \\
& \leq \sum_{N+1}^{M}\left[\frac{(n+1)^{\gamma}-n^{\gamma}}{n^{2 \gamma}}\right] \frac{n C_{f}}{n^{\beta}}+\frac{M C_{f}}{M^{\gamma} M^{\beta}}+\frac{N C_{f}}{N^{\gamma} N^{\beta}} \\
& \leq \sum_{N+1}^{M} \frac{C_{f}\left[(n+1)^{\gamma}-n^{\gamma}\right]}{n^{2 \gamma+\beta-1}}+\frac{C_{f}}{M^{\gamma+\beta-1}}+\frac{C_{f}}{N^{\gamma+\text { beta-1 }}} .
\end{aligned}
$$

Hence,

$$
\left\|\sup _{\varepsilon}\left|S_{M}^{\varepsilon}(f)-S_{N}^{\varepsilon}(f)\right|\right\|_{1} \leq \sum_{N+1}^{M} \frac{C_{f}}{n^{\gamma+\beta}}+\frac{C_{f}}{N^{\gamma+\beta-1}} .
$$

Substituting $2^{N+1}$ for $M$ and $2^{N}$ for $N$ we have the following.

$$
\left\|\sup _{\varepsilon}\left|S_{2^{N+1}}^{\varepsilon}(f)-S_{2^{N}}^{\varepsilon}(f)\right|\right\|_{1} \leq \sum_{2^{N}+1}^{2^{N+1}} \frac{C_{f}}{n^{\gamma+\beta}}+\frac{C_{f}}{\left(2^{\gamma+\beta-1}\right)^{N}} .
$$

Since $\gamma+\beta>\gamma+\beta / 2>1, \sum_{N=1}^{\infty}\left\|\sup _{\varepsilon}\left|S_{2^{N+1}}^{\varepsilon}(f)-S_{2^{N}}^{\varepsilon}(f)\right|\right\|_{1}<\infty$. Hence, $\sum_{N=1}^{\infty} \sup _{\varepsilon}\left|S_{2^{N+1}}^{\varepsilon}(f)-S_{2^{N}}^{\varepsilon}(f)\right|<\infty$ a.e., i.e. there exists a set $X_{f}$ of full 
measure such that

$$
\sum_{N=1}^{\infty} \sup _{\varepsilon}\left|S_{2^{N+1}}^{\varepsilon}(f)(x)-S_{2^{N}}^{\varepsilon}(f)(x)\right|<\infty \quad \forall x \in X_{f} .
$$

From this we can conclude that $S_{2^{N}}^{\varepsilon}(f)$ converges a.e. to a continuous function of $\varepsilon$. Furthermore, from (7) we get that

$$
\begin{array}{r}
\sup _{2^{N} \leq L \leq 2^{N+1} \sup _{\varepsilon}\left|S_{L}^{\varepsilon}(f)-S_{2^{N}}^{\varepsilon}(f)\right| \leq \sum_{2^{N}+1}^{2^{N+1}-1}}\left[\frac{1}{n^{\gamma}}-\frac{1}{(n+1)^{\gamma}}\right] \sup _{\varepsilon}\left|n M_{n}^{\varepsilon}(f)\right| \\
+2 \sup _{2^{N} \leq L \leq 2^{N+1}} \sup _{\varepsilon}\left|\frac{L M_{L}^{\varepsilon}(f)}{L^{\gamma}}\right| .
\end{array}
$$

The first term goes to zero a.e. as $\mathrm{N} \rightarrow \infty$ since

$$
\begin{aligned}
\sum_{N=1}^{\infty}\left\|\sum_{2^{N}+1}^{2^{N+1}-1}\left[\frac{1}{n^{\gamma}}-\frac{1}{(n+1)^{\gamma}}\right] \sup _{\varepsilon}\left|n M_{n}^{\varepsilon}(f)\right|\right\|_{1} & \leq \sum_{N=1}^{\infty} \sum_{2^{N}+1}^{2^{N+1}-1} \frac{\gamma}{n^{2 \gamma} n^{1-\gamma}} \frac{n C_{f}}{n^{\beta}} \\
& =\sum_{N=1}^{\infty} \frac{C_{f}}{n^{\gamma+\beta}}<\infty
\end{aligned}
$$

since $\gamma+\beta>1$.

To finish the proof of the theorem, it only remains to show that the last term goes to 0 a.e. as $N \rightarrow \infty$.

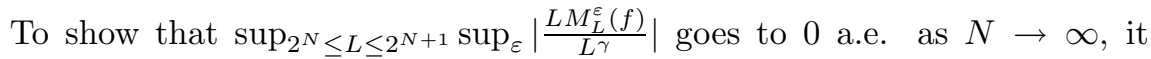
suffices to show that

$$
R_{L}=\sup _{\varepsilon}\left|\frac{L M_{L}^{\varepsilon}(f)}{L^{\gamma}}\right| \longrightarrow 0 \text { a.e. as } \mathrm{L} \longrightarrow \infty \text {. }
$$

First, let us look at the $L^{1}$ norm of $R_{L}$.

$$
\left\|R_{L}\right\|_{1}=\left\|\sup _{\varepsilon} \mid \frac{L M_{L}^{\varepsilon}(f)}{L^{\gamma}}\right\| \|_{1} \leq \frac{L C_{f}}{L^{\gamma} L^{\beta}}=\frac{C_{f}}{L^{\gamma+\beta-1}}
$$

where the inequality follows by assumption.

Let $\rho=\gamma+\beta-1$ and note that $\rho>0$ since $\gamma>1-\beta / 2>1-\beta>0$.

Now let $L=\left[N^{\delta}\right]$ where $\delta$ is such that $1 / \rho<\delta<1 /\left(1+\frac{1}{p}-\gamma\right)$. It is possible to pick such a $\delta$ since by assumption $\gamma>1+\frac{1}{2 p}-\frac{\beta}{2}$. Also note that $\rho=(\gamma-1)+\beta<\beta<1$ implies that $\delta>1$. Then,

$$
\sum_{N=1}^{\infty}\left\|R_{\left[N^{\delta}\right]}\right\|_{1} \leq \sum_{N=1}^{\infty} \frac{C_{f}}{\left[N^{\delta}\right]^{\rho}}<\infty
$$

since $\rho \delta>1$.

BULLETIN DE LA SOCIÉTÉ MATHÉMATIQUE DE FRANCE 
Hence, $R_{\left[N^{\delta}\right]} \rightarrow 0$ a.e. That is,

$$
\sup _{\varepsilon}\left|\frac{1}{\left[N^{\delta}\right]^{\gamma}} \sum_{n=1}^{\left[N^{\delta}\right]} f\left(T^{n} x\right) e^{2 \pi i n \varepsilon}\right| \longrightarrow 0 \text { a.e. as } N \rightarrow \infty .
$$

Now, let $\left[N^{\delta}\right] \leq L \leq\left[(N+1)^{\delta}\right]$. Then,

$$
\begin{aligned}
\sup _{\varepsilon} \mid \frac{1}{L^{\gamma}} & \sum_{n=1}^{L} f\left(T^{n} x\right) e^{2 \pi i n \varepsilon} \mid \\
& \leq \sup _{\varepsilon}\left|\frac{1}{\left[N^{\delta}\right]^{\gamma}} \sum_{n=1}^{\left[N^{\delta}\right]} f\left(T^{n} x\right) e^{2 \pi i n \varepsilon}\right|+\sup _{\varepsilon}\left|\frac{1}{L^{\gamma}} \sum_{n=\left[N^{\delta}\right]+1}^{L} f\left(T^{n} x\right) e^{2 \pi i n \varepsilon}\right| .
\end{aligned}
$$

The first term goes to zero a.e. by (8). The second term also goes to zero a.e. since

$$
\begin{aligned}
\sup _{\varepsilon}\left|\frac{1}{L^{\gamma}} \sum_{n=\left[N^{\delta}\right]+1}^{L} f\left(T^{n} x\right) e^{2 \pi i n \varepsilon}\right| & \leq \frac{1}{L^{\gamma}} \sum_{n=\left[N^{\delta}\right]+1}^{\left[(N+1)^{\delta}\right]}\left|f\left(T^{n} x\right)\right| \\
& \leq \frac{1}{\left[N^{\delta}\right]^{\gamma}} \sum_{n=\left[N^{\delta}\right]+1}^{\left[(N+1)^{\delta}\right]}\left|f\left(T^{n} x\right)\right| \longrightarrow 0 \text { a.e. }
\end{aligned}
$$

by the proposition.

\section{REMARKS}

(1) There exist $\gamma \in(0,1)$ for which the above theorem is not true. In particular, if $\left\{X_{n}\right\}$ is the Rademacher sequence, then the map $\varepsilon \mapsto \sum_{n=1}^{\infty} \frac{X_{n}(\omega) e^{2 \pi i n \varepsilon}}{n^{\gamma}}$ is not continuous if $\gamma \leq 1 / 2$ since

$$
\begin{aligned}
\sup _{\varepsilon \in[0,1]}\left|\sum_{n=1}^{\infty} \frac{X_{n}(\omega) e^{2 \pi i n \varepsilon}}{n^{\gamma}}\right|^{2} & \geq \int_{0}^{1}\left|\sum_{n=1}^{\infty} \frac{X_{n}(\omega) e^{2 \pi i n \varepsilon}}{n^{\gamma}}\right|^{2} d \varepsilon \\
& =\sum_{n=1}^{\infty} \frac{\left|X_{n}(\omega)\right|^{2}}{n^{2 \gamma}} \\
& =\sum_{n=1}^{\infty} \frac{1}{n^{2 \gamma}}=\infty .
\end{aligned}
$$

As pointed out by the referee this argument can be extended to ergodic stationary processes in the following way:

TOME $129-2001-\mathrm{N}^{\mathrm{O}} 3$ 
If the series $\sum_{n=1}^{\infty} \frac{f\left(T^{n} x\right) e^{2 \pi i n \varepsilon}}{n^{1 / 2}}$ converges uniformly in $\varepsilon$ for a.e. $x$, then

$$
\begin{aligned}
\sum_{n=1}^{\infty} \frac{\left|f\left(T^{n} x\right)\right|^{2}}{n} & =\int_{0}^{1}\left|\sum_{n=1}^{\infty} \frac{f\left(T^{n} x\right) e^{2 \pi i n \varepsilon}}{n^{1 / 2}}\right|^{2} d \varepsilon \\
& \leq \sup _{\varepsilon \in[0,1]}\left|\sum_{n=1}^{\infty} \frac{f\left(T^{n} x\right) e^{2 \pi i n \varepsilon}}{n^{1 / 2}}\right|^{2}<\infty
\end{aligned}
$$

which contradicts the fact that

$$
\lim _{N \rightarrow \infty} \frac{1}{\log N} \sum_{n=1}^{N} \frac{\left|f\left(T^{n} x\right)\right|^{2}}{n}=\int|f|^{2} d \mu .
$$

(2) In [2], Theorem 3 is used to give a spectral characterization of $L^{\infty}$ Wiener-Wintner functions of some power type in $L^{2}$.

\section{BIBLIOGRAPHY}

[1] Assani (I.) - Wiener-wintner dynamical systems, Preprint, 1998.

[2] _ Spectral characterization of Wiener-Wintner dynamical systems, Prépublication IRMA Strasbourg, June 2000.

[3] Bourgain (J.) - Double recurrence and almost sure convergence, J. reine angew. Math., t. 404 (1990), pp. 140-161.

[4] Furstenberg (H.) - Recurrence in ergodic theory and combinatorial number theory, Princeton University Press, Princeton, NJ, 1981.

[5] IWANiK (A.), LEMANCZYK (M.) \& MAUduit (C.) - Spectral properties of piecewise absolutely continuous cocycles over irrational rotations, J. London Math. Soc. (2), t. 59 (1996), pp. 171-187.

[6] Khinchin (A.) - Einige Sätze über Kettenbrüche, mit Anwendungen auf die Theorie der Diophantischen Approximationen, Math. Ann., t. 92 (1924), pp. 115-125.

[7] _ Continued fractions, The University of Chicago press, Chicago, Illinois, 1964

[8] Kuipers (L.) \& NiederReiter (H.) - Uniform distribution of sequences, John Wiley and Sons, 1974.

[9] Medina (H.) - Spectral Types of Unitary Operators Arising from Irrational Rotations on the Circle Group, Michigan Math. J., t. 41 (1994), pp. 39-49.

[10] Schwartz (M.) - Polynomially moving ergodic averages, Proc. Amer. Math. Soc., t. 103 (1988), no. 1, pp. 252-254. 